\title{
BORDISM CLASSES OF VECTOR BUNDLES OVER REAL PROJECTIVE SPACES
}

\author{
BRUCE F. TORRENCE
}

(Communicated by Frederick R. Cohen)

\begin{abstract}
A basis is presented for the $\mathbf{R P}^{n}$ classes in $\mathfrak{N}_{n}(B O)$. This basis is used to prove that every smooth involution $(M, T)$ on a closed manifold bounds if its fixed point set is a disjoint union of odd-dimensional projective spaces of constant dimension.
\end{abstract}

Given any real vector bundle $\xi^{c} \rightarrow \mathbf{R P}^{n}$, let $\left(\mathbf{R P}^{n}, \xi\right) \in \mathfrak{N}_{n}(B O)$ denote the stable bordism class determined by the map $\mathbf{R P}^{n} \rightarrow B O(c) \hookrightarrow B O$ classifying $\xi$. With $n$ fixed, the classes $\left(\mathbf{R P}^{n}, \xi\right)$ as $\xi$ ranges over all vector bundles span a $\mathbf{Z}_{2}$-vector subspace of $\mathfrak{N}_{n}(B O)$, where the sum $\left(\mathbf{R P}^{n}, \xi\right)+\left(\mathbf{R P}^{n}, \eta\right)$ represents the disjoint union. We will exhibit a basis for this subspace.

Letting $\lambda \rightarrow \mathbf{R P}^{n}$ denote the canonical line bundle, the KO-theory of real vector bundles tells us that any $\xi \rightarrow \mathbf{R P}^{n}$ is stably isomorphic to $k \lambda$ for some (possibly large) $k$, so that $\left(\mathbf{R P}^{n}, \xi\right)=\left(\mathbf{R P}^{n}, k \lambda\right)$. The main result of this paper is that up to bordism one need only consider those $k \leq n$.

Theorem. A basis for the $\mathbf{R P}^{n}$ classes in $\mathfrak{N}_{n}(B O)$ is

$$
\begin{array}{ll}
\left\{\left(\mathbf{R P}^{n}, j \lambda\right) \mid 0 \leq j \leq n\right\} & \text { if } n=2 m, \\
\left\{\left(\mathbf{R P}^{n},(2 j+1) \lambda\right) \mid 0 \leq j \leq m\right\} & \text { if } n=2 m+1 .
\end{array}
$$

Thus, given a bundle $k \lambda \rightarrow \mathbf{R P}^{n}$ with $k>n$, the theorem tells us that

$$
\left(\mathbf{R P}^{n}, k \lambda\right)=\sum_{j}\left(\mathbf{R P}^{n}, j \lambda\right)
$$

with each $j \leq n$. As an application we include the following

Proposition. Given a closed manifold with differentiable involution $(M, T)$ with fixed point set any disjoint union $F=\bigcup \mathbf{R P}^{2 n+1}$ ( $n$ fixed), then $(M, T)$ bounds.

Capobianco [1] has shown this is true when $F=\mathbf{R P}^{2 m+1}$. Royster [4] proved it when $F=\mathbf{R P}^{2 n+1} \cup \mathbf{R P}^{2 m+1}$. No such result is possible for $F=\bigcup \mathbf{R} \mathbf{P}^{2 n}$. Indeed, Stong [5] characterized all involutions fixing $\mathbf{R P}^{2 n}$. The author wishes

Received by the editors October 29, 1991.

1991 Mathematics Subject Classification. Primary 55N22, 57R90, 55R15.

Key words and phrases. Cobordism, involution. 
to express his gratitude and indebtedness to Professor Stong, whose insight and generous counsel made this work possible.

\section{PROOF OF THE THEOREM}

Let $\left[\mathbf{R P}{ }^{n}\right] \in H_{n}\left(\mathbf{R P}^{n} ; \mathbf{Z}_{2}\right)$ denote the fundamental class. Let $\tau \rightarrow \mathbf{R P}^{n}$ denote the tangent bundle. The bordism class $\left(\mathbf{R P}^{n}, \xi\right)$ is completely determined by the characteristic numbers

$$
\left\langle w_{I}(\xi) w_{J}(\tau) ;\left[\mathbf{R} \mathbf{P}^{n}\right]\right\rangle \in \mathbf{Z}_{2},
$$

where the union $I \cup J$ is a partition of $n$.

We show first that the classes $\left(\mathbf{R} \mathbf{P}^{n}, j \lambda\right)$ of the theorem are linearly independent. Let $\alpha \in H^{1}\left(\mathbf{R P}^{n} ; \mathbf{Z}_{2}\right)$ denote the nonzero class. For $n=2 m$ consider the $(n+1) \times(n+1)$ matrix whose $(i+1, j+1)$ st entry is the characteristic number

$$
\left\langle w_{i}(j \lambda) w_{1}(\tau)^{n-i} ;\left[\mathbf{R P}^{n}\right]\right\rangle=\left\langle\left(\begin{array}{c}
j \\
i
\end{array}\right) \alpha^{i} \alpha^{n-i} ;\left[\mathbf{R P}^{n}\right]\right\rangle=\left(\begin{array}{c}
j \\
i
\end{array}\right) \quad(\bmod 2) .
$$

This matrix is upper triangular with each diagonal entry equal to 1 , so its columns are linearly independent. Thus the classes $\left\{\left(\mathbf{R P}^{n}, j \lambda\right) \mid 0 \leq j \leq n\right\}$ are linearly independent.

For $n=2 m+1$, one must show that the classes $\left\{\left(\mathbf{R P}^{n} ;(2 j+1) \lambda\right) \mid 0 \leq j \leq m\right\}$ are linearly independent. One argues as above, but uses the $(m+1) \times(m+1)$ matrix with $(i+1, j+1)$ st entry

$$
\left\langle w_{2 i+1}((2 j+1) \lambda) w_{1}((2 j+1) \lambda)^{n-(2 i+1)} ;\left[\mathbf{R} \mathbf{P}^{n}\right]\right\rangle .
$$

Note that for $n$ odd, the classes $\left(\mathbf{R P}^{n}, k \lambda\right)$ with $k$ even are boundaries, as all nonzero classes $w_{I}(k \lambda) w_{J}(\tau)$ are even powers of $\alpha$, so that all characteristic numbers vanish.

We now must show that for any $n$, the classes $\left\{\left(\mathbf{R P}^{n}, j \lambda\right) \mid j \leq n\right\}$ span the $\mathbf{R P}^{n}$ classes in $\mathfrak{N}_{n}(B O)$. The KO-theory of real bundles tells us that any class $\left(\mathbf{R} \mathbf{P}^{n}, \xi\right)$ is bordant to $\left(\mathbf{R} \mathbf{P}^{n}, k \lambda\right)$ for some (possibly large) $k$. If $n=0$ the theorem is trivial. If $2^{a} \leq n<2^{a+1}$, we may assume that $k<2^{a+1}$ since the total Stiefel-Whitney class $w\left(2^{a+1} \lambda\right)=(1+\alpha)^{2^{a+1}}=1$. We will show that any class $\left(\mathbf{R P}^{n}, k \lambda\right)$ with $2^{a} \leq n<k<2^{a+1}$ is bordant to a disjoint union $\sum_{j}\left(\mathbf{R P}^{n}, j \lambda\right)$ with $j \leq n$. We first exhibit a decomposition $w(k \lambda)=\sum_{j} w(j \lambda)$ with each $j \leq n$.

Lemma 1. Let $2^{a} \leq n<k<2^{a+1}$. In the ring $\mathbf{Z}_{2}[\alpha] /\left(\alpha^{n+1}\right)=H^{*}\left(\mathbf{R P}^{n} ; \mathbf{Z}_{2}\right)$, one has a unique decomposition

$$
(1+\alpha)^{k}=(1+\alpha)^{k-2^{a}}+\sum_{t}\left[(1+\alpha)^{j_{t}}+(1+\alpha)^{2^{a}+j_{t}}\right]
$$

with $0 \leq j_{t} \leq n-2^{a}$.

Proof. A $\mathbf{Z}_{2}$ basis for $\mathbf{Z}_{2}[\alpha] /\left(\alpha^{n+1}\right)$ is $\left\{1, \alpha, \ldots, \alpha^{n}\right\}$. Since an upper triangular matrix with diagonal entries equal to 1 maps $\alpha^{i} \mapsto(1+\alpha)^{i}, 0 \leq i \leq n$, it follows that $\left\{1,(1+\alpha), \ldots,(1+\alpha)^{n}\right\}$ is also a basis. Now

$$
(1+\alpha)^{k}=(1+\alpha)^{k-2^{a}}+\alpha^{2^{a}}(1+\alpha)^{k-2^{a}} .
$$


Notice that in the rightmost summand each power of $\alpha$ in $(1+\alpha)^{k-2^{a}}$ with exponent greater than $n-2^{a}$ is killed by $\alpha^{2^{a}}$. By the preceding comment, there is a unique decomposition

$$
(1+\alpha)^{k-2^{a}}=\sum_{t}(1+\alpha)^{j_{t}}
$$

in the ring $\mathbf{Z}_{2}[\alpha] /\left(\alpha^{n-2^{a}+1}\right)$, with $0 \leq j_{t} \leq n-2^{a}$ for each $t$. Thus

$$
\begin{aligned}
(1+\alpha)^{k} & =(1+\alpha)^{k-2^{a}}+\alpha^{2^{a}}\left[\sum_{t}(1+\alpha)^{j_{t}}\right] \\
& =(1+\alpha)^{k-2^{a}}+\left(1+1+\alpha^{2^{a}}\right)\left[\sum_{t}(1+\alpha)^{j_{t}}\right] \\
& =(1+\alpha)^{k-2^{a}}+\left[\sum_{t}(1+\alpha)^{j_{t}}+(1+\alpha)^{2^{a}+j_{t}}\right] .
\end{aligned}
$$

There is a useful case where the decomposition has exactly three summands. Suppose $n>2^{a}$, so there is a $0 \leq b<a$ with $2^{b} \leq n-2^{a}<2^{b+1}$. Let $0 \leq k^{\prime}<2^{b+1}$ satisfy $k^{\prime} \equiv k \equiv k-2^{a}\left(\bmod 2^{b+1}\right)$, so that

$$
(1+\alpha)^{k}=(1+\alpha)^{k-2^{a}}+\alpha^{2^{a}}(1+\alpha)^{k^{\prime}} \text {. }
$$

If $k^{\prime} \leq n-2^{a}$ then

$$
(1+\alpha)^{k}=(1+\alpha)^{k-2^{a}}+\left[(1+\alpha)^{k^{\prime}}+(1+\alpha)^{2^{a}+k^{\prime}}\right] .
$$

The following lemma will complete the proof of the theorem:

Lemma 2. Let $2^{a} \leq n<k<2^{a+1}$. The class $\left(\mathbf{R P}^{n}, k \lambda\right) \in \mathfrak{N}_{n}(B O)$ may be uniquely expressed as a disjoint union

$$
\left(\mathbf{R P}^{n}, k \lambda\right)=\left(\mathbf{R P}^{n},\left(k-2^{a}\right) \lambda\right)+\sum_{t}\left[\left(\mathbf{R P}^{n}, j_{t} \lambda\right)+\left(\mathbf{R P}^{n},\left(2^{a}+j_{t}\right) \lambda\right)\right]
$$

with the $j_{t}$ as in Lemma 1.

Proof. Uniqueness follows from the linear independence of the classes on the right-hand side. To show for each partition $I$ and $J$ with union $I \cup J$ a partition of $n$ that

$$
\begin{aligned}
\left\langle w_{I}(k \lambda) w_{J}(\tau) ;\left[\mathbf{R} \mathbf{P}^{n}\right]\right\rangle= & \left\langle w_{I}\left(\left(k-2^{a}\right) \lambda\right) w_{J}(\tau) ;\left[\mathbf{R} \mathbf{P}^{n}\right]\right\rangle \\
& +\sum_{t}\left[\left\langle w_{I}\left(j_{t} \lambda\right) w_{J}(\tau) ;\left[\mathbf{R} \mathbf{P}^{n}\right]\right\rangle\right. \\
& \left.+\left\langle w_{I}\left(\left(2^{a}+j_{t}\right) \lambda\right) w_{J}(\tau) ;\left[\mathbf{R P}^{n}\right]\right\rangle\right],
\end{aligned}
$$

it suffices to show that for each partition $I=i_{1}, i_{2}, \ldots, i_{r}$ with $|I|=\sum i \leq n$ one has

$$
w_{I}(k \lambda)=w_{I}\left(\left(k-2^{a}\right) \lambda\right)+\sum_{t}\left[w_{I}\left(j_{t} \lambda\right)+w_{I}\left(\left(2^{a}+j_{t}\right) \lambda\right)\right]
$$

Notice that Lemma 1 tells us that the total Stiefel-Whitney classes satisfy the equation

$$
w(k \lambda)=w\left(\left(k-2^{a}\right) \lambda\right)+\sum_{t}\left[w\left(j_{t} \lambda\right)+w\left(\left(2^{a}+j_{t}\right) \lambda\right)\right]
$$

In particular, if $I=i_{1}$ then the result holds. 
Case 1. Each $i_{s}<2^{a}$. In this case we have $w_{i_{s}}(k \lambda)=w_{i_{s}}\left(\left(k-2^{a}\right) \lambda\right)$ and, for each $t, w_{i_{s}}\left(j_{t} \lambda\right)=w_{i_{s}}\left(\left(2^{a}+j_{t}\right) \lambda\right)$. Hence the product $w_{I}(k \lambda)=w_{I}\left(\left(k-2^{a}\right) \lambda\right)$ and, for each $t, w_{I}\left(j_{t} \lambda\right)=w_{I}\left(\left(2^{a}+j_{t}\right) \lambda\right)$, and the lemma holds since we are working over $\mathbf{Z}_{2}$.

Case 2. $i_{1} \geq 2^{a}$. Note that no more than one of the $i_{s}$ may be greater than or equal to $2^{a}$ since $\sum i \leq n<2^{a+1}$. We assume without loss of generality that $i_{1} \geq 2^{a}$. Note also that since each $j_{t}<2^{a}$ and $k-2^{a}<2^{a}$, we have $w_{I}\left(j_{t} \lambda\right)=w_{I}\left(\left(k-2^{a}\right) \lambda\right)=0$. So we must show

$$
w_{I}(k \lambda)=\sum_{t} w_{I}\left(\left(2^{a}+j_{t}\right) \lambda\right) .
$$

If $n=2^{a}$, then $2^{a} \leq i_{1} \leq|I| \leq n=2^{a}$, so that $I=i_{1}=2^{a}$, and the result holds. In particular, if $a=1$, then $n=2=2^{a}$ and $k=3$, and the lemma is true. We proceed with the assumption that $n>2^{a}$ and that $I$ has length at least 2 and use induction on $a$.

Let $0 \leq b<a$ satisfy $2^{b} \leq n-2^{a}<2^{b+1}$, and let $0 \leq k^{\prime}<2^{b+1}$ satisfy $k^{\prime} \equiv k \equiv k-2^{a}\left(\bmod 2^{b+1}\right)$. Let $K=i_{1}-2^{a}, i_{2}, \ldots, i_{r}$. Observe that $|K| \leq n-2^{a}$ so that

$$
w_{i_{s}}(k \lambda)=w_{i_{s}}\left(k^{\prime} \lambda\right)=w_{i_{s}}\left(\left(2^{a}+k^{\prime}\right) \lambda\right)
$$

for $2 \leq s \leq r$ and

$$
w_{i_{1}}(k \lambda)=\alpha^{2^{a}} w_{i_{1}-2^{a}}\left(k^{\prime} \lambda\right)=\alpha^{2^{a}} w_{i_{1}-2^{a}}\left(\left(2^{a}+k^{\prime}\right) \lambda\right) .
$$

If $k^{\prime} \leq n-2^{a}$, then we have

$$
w_{I}(k \lambda)=\alpha^{2^{a}} w_{K}\left(k^{\prime} \lambda\right)=w_{I}\left(\left(2^{a}+k^{\prime}\right) \lambda\right) .
$$

By (2) this last equation is equation (4), and we are done.

If $k^{\prime}>n-2^{a}$, write the total class $w\left(k^{\prime} \lambda\right)=w\left(\left(k-2^{a}\right) \lambda\right)=\sum_{t} w\left(j_{t} \lambda\right)$ as in (1). Then

for each $t$ and

$$
w_{i_{1}}\left(\left(2^{a}+j_{t}\right) \lambda\right)=\alpha^{2^{a}} w_{i_{1}-2^{a}}\left(j_{t} \lambda\right)
$$

$$
w_{i_{s}}\left(\left(2^{a}+j_{t}\right) \lambda\right)=w_{i_{s}}\left(j_{t} \lambda\right)
$$

for $2 \leq s \leq r$. Now invoking the inductive hypothesis with $2^{b} \leq n-2^{a}<k^{\prime}<$ $2^{b+1}$, we may assume that $(3)$ holds for $K$, that is, $w_{K}\left(k^{\prime} \lambda\right)=\sum_{t} w_{K}\left(j_{t} \lambda\right)$. But then

$$
\begin{aligned}
w_{I}(k \lambda) & =\alpha^{2^{a}} w_{K}\left(k^{\prime} \lambda\right)=\alpha^{2^{a}} \sum_{t} w_{K}\left(j_{t} \lambda\right) \\
& =\sum_{t} \alpha^{2^{a}} w_{K}\left(j_{t} \lambda\right)=\sum_{t} w_{I}\left(\left(2^{a}+j_{t}\right) \lambda\right),
\end{aligned}
$$

and equation (4) holds.

\section{PROOF OF THE PROPOSITION}

Suppose we are given a closed manifold with differentiable involution $\left(M^{m}, T\right)$ with fixed point set $F=\bigcup \mathbf{R P}^{2 n-1}$, where $n \geq 1$ is fixed. We wish to show that the involution $(M, T)$ bounds. Note that if $m=2 n-1$ then the proposition is trivial, as $F$ consists of connected components of $M$ 
each of which bounds in its own right, and $T$ acts freely on the components of $M-F$. So assume that $m>2 n-1$. In [2] Conner and Floyd showed that the fixed point structure of a differentiable involution on a closed manifold determines the bordism class. That is, if $\mathfrak{N}_{m}^{\mathbf{Z}_{2}}$ denotes the group of unoriented bordism classes of involutions on $m$-manifolds, then the composition

$$
\mathfrak{N}_{m}^{\mathbf{Z}_{2}} \rightarrow \sum_{k=0}^{m} \mathfrak{N}_{k}(\mathbf{B O}(m-k)) \rightarrow \sum_{k=0}^{m} \mathfrak{N}_{k}(\mathbf{B O})
$$

is monic, where the first map assigns to each involution its fixed point data and the second is induced by the inclusion $\mathbf{B O}(m-k) \hookrightarrow \mathbf{B O}$. Let $\nu \rightarrow F$ denote the normal bundle of $F$ in $M$. Then either $(F, \nu)=0 \in \mathfrak{N}_{2 n-1}(\mathbf{B O})$ and $(M, T)$ bounds, or according to the theorem we may write

$$
(F, \nu)=\sum_{i}\left(\mathbf{R} \mathbf{P}^{2 n-1}, \nu_{i}\right)=\sum_{i}\left(\mathbf{R P}^{2 n-1},\left(2 k_{i}-1\right) \lambda\right)
$$

with $1 \leq k_{i} \leq n$ for each $i$ and $k_{i} \neq k_{j}$ for $i \neq j$. We will show that the latter is impossible.

So suppose $(F, \nu)=\sum_{i}\left(\mathbf{R P}^{2 n-1},\left(2 k_{i}-1\right) \lambda\right)$ as above. Then note that each $2 k_{i}-1$ is no greater than the codimension $m-(2 n-1)$ for

$$
w_{2 k_{i}-1}\left(\nu_{i}^{m-(2 n-1)}\right)=w_{2 k_{i}-1}\left(\left(2 k_{i}-1\right) \lambda\right)=\alpha^{2 k_{i}-1} \neq 0 .
$$

According to Kosniowski and Stong [3], if $f\left(x_{1}, x_{2}, \ldots, x_{m}\right)$ is any symmetric polynomial over $\mathbf{Z}_{2}$ in $m$ variables of degree at most $m$, then

$$
\begin{aligned}
f\left(x_{1},\right. & \left.x_{2}, \ldots, x_{m}\right)\left[M^{m}\right] \\
\quad= & \sum_{i} \frac{f\left(1+y_{1}, 1+y_{2}, \ldots, 1+y_{m-(2 n-1)}, z_{1}, z_{2}, \ldots, z_{2 n-1}\right)}{\left(1+y_{1}\right)\left(1+y_{2}\right) \cdots\left(1+y_{m-(2 n-1)}\right)}\left[\mathbf{R P}^{2 n-1}\right]
\end{aligned}
$$

where $i$ is as above, indexing copies of $\mathbf{R P}^{2 n-1}$ in $F$, and where the expressions are evaluated by replacing the elementary symmetric functions $\sigma_{j}(x)$, $\sigma_{j}(y)$, and $\sigma_{j}(z)$ by the Stiefel-Whitney classes $w_{j}(M), w_{j}\left(\left(2 k_{i}-1\right) \lambda\right)$, and $w_{j}\left(\mathbf{R P}^{2 n-1}\right)$, respectively and evaluating the resulting cohomology class on the fundamental homology class of $M^{m}$ or $\mathbf{R P}^{2 n-1}$. In our case, since each $2 k_{i}-1 \leq m-(2 n-1)$, the expression $\left(1+y_{1}\right)\left(1+y_{2}\right) \cdots\left(1+y_{m-(2 n-1)}\right)$ in each denominator on the right is equal to the total class $w\left(\left(2 k_{i}-1\right) \lambda\right)=(1+\alpha)^{2 k_{i}-1}$.

Let $f\left(x_{1}, \ldots, x_{m}\right)=x_{1}+x_{2}+\cdots+x_{m}$. Then for each $i$

$$
\begin{aligned}
f\left(1+y_{1}, \ldots, z_{2 n-1}\right) & =(m-(2 n-1))+w_{1}\left(\left(2 k_{i}-1\right) \lambda\right)+w_{1}\left(\mathbf{R P}^{2 n-1}\right) \\
& =(m+1)+\left(\begin{array}{c}
2 k_{i}-1 \\
1
\end{array}\right) \alpha+\left(\begin{array}{c}
2 n \\
1
\end{array}\right) \alpha \\
& =(m+1)+\alpha .
\end{aligned}
$$

Now for $1 \leq j \leq n$ let

$$
f_{j}\left(x_{1}, \ldots, x_{m}\right)=\left(f\left(x_{1}, \ldots, x_{m}\right)+(m+1)\right)^{2 j-1} .
$$

Then for each $j$ we have $f_{j}\left(x_{1}, \ldots, x_{m}\right)[M]=0$ since no terms in $f_{j}$ have degree greater than $2 j-1 \leq 2 n-1<m$. On the other hand, for each $i$, 
$f_{j}\left(1+y_{1}, \ldots, z_{2 n-1}\right)=((m+1)+(m+1)+\alpha)^{2 j-1}=\alpha^{2 j-1}$. So for each $1 \leq j \leq n$ we have

$$
0=\sum_{i} \frac{\alpha^{2 j-1}}{(1+\alpha)^{2 k_{i}-1}}\left[\mathbf{R P}^{2 n-1}\right] .
$$

To evaluate this sum, note that for any $k>0$,

$$
\frac{1}{(1+\alpha)^{k}}=\sum_{t=0}^{2 n-1}\left(\begin{array}{c}
k-1+t \\
t
\end{array}\right) \alpha^{t}
$$

in the ring $H^{*}\left(\mathbf{R} \mathbf{P}^{2 n-1} ; \mathbf{Z}_{2}\right)$, as is seen by induction on $k$. Hence we have for each $j$

$$
\begin{aligned}
0 & =\sum_{i} \frac{\alpha^{2 j-1}}{(1+\alpha)^{2 k_{i}-1}}\left[\mathbf{R P}^{2 n-1}\right] \\
& =\sum_{i}\left(\begin{array}{c}
\left(2 k_{i}-1\right)-1+(2 n-1)-(2 j-1) \\
(2 n-1)-(2 j-1)
\end{array}\right) \\
& =\sum_{i}\left(\begin{array}{c}
k_{i}-1+(n-j) \\
(n-j)
\end{array}\right)(\bmod 2) .
\end{aligned}
$$

But this is impossible. Let $A_{n}$ be the $n \times n$ matrix over $\mathbf{Z}_{2}$ with $(j, k)$ th entry $\left(\begin{array}{c}k-1+(n-j) \\ (n-j)\end{array}\right)$. Then

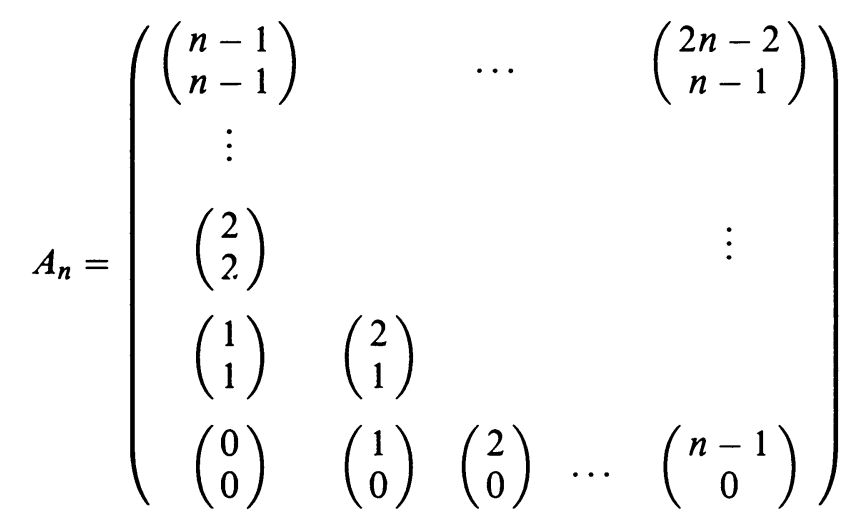

and $A_{n}$ is the "upper corner" of Pascal's triangle reduced modulo 2. If $n=2^{a}$, then $A_{n}$ is lower triangular with each diagonal entry equal to 1 , so $A_{n}$ is nonsingular. If $2^{a}<n<2^{a+1}$, then after a finite sequence of elementary row and column operations $A_{n}$ may be reduced to

$$
\left(\begin{array}{cc}
0 & A_{n-2^{a}} \\
A_{2^{a}} & 0
\end{array}\right)
$$

and so is nonsingular by induction. In particular, the columns of $A_{n}$ are linearly independent, so that the sum (5) cannot be 0 for every $j$ with $1 \leq j \leq n$. This is a contradiction. We conclude that $(F, \nu)=0 \in \mathfrak{N}_{2 n-1}(\mathbf{B O})$ so that $(M, T)$ bounds. 


\section{REFERENCES}

1. F. L. Capobianco, Stationary points of $\left(Z_{2}\right)^{k}$-actions, Proc. Amer. Math. Soc. 67 (1976), 377-380.

2. P. E. Conner and E. E. Floyd, Differentiable periodic maps, Springer-Verlag, Berlin, 1964.

3. C. Kosniowski and R. E. Stong, Involutions and characteristic numbers, Topology 17 (1978), 309-330.

4. D. C. Royster, Involutions fuxing the disjoint union of two projective spaces, Indiana Univ. Math. J. 29 (1980), 267-276.

5. R. E. Stong, Involutions fixing projective spaces, Michigan Math. J. 13 (1966), 445-457.

Department of Mathematics, Georgetown University, Washington, District of Columbia 20057-0996

E-mail address: bruce@gumath1.georgetown.edu 